\title{
Designing babies through gene editing: science or science fiction?
}

\author{
A. Cecile J.W. Janssens ${ }^{1,2}$
}

Recently, an international group of experts met in Washington, DC, to discuss scientific, ethical, and governance issues regarding gene editing research and applications. The summit was convened following the rapid adoption of the CRISPR (clustered regularly interspaced short palindromic repeat)-Cas9 gene editing technology, which is already being used by laboratories around the world to make cells and breed laboratory animals with modified DNA for the study of diseases. ${ }^{1,2}$ CRISPR offers entirely new opportunities for the study of the function of genes in the development and progression of human disease and for the development of human gene therapy, stem cell therapy, and the culture of human tissue, ${ }^{3,4}$ but is not without risks.

Scientists and bioethicists are concerned that gene editing opens Pandora's box, ${ }^{4,5}$ warning that imperfections of the technology may introduce errors such as off-target cuts that cause heritable modifications and that even successful edits may have disastrous consequences, including irreversible damage to the health of individuals, assaults on human dignity, issues stemming from justice inequality, and unpredictable and unknown threats to entire populations and future generations., ${ }^{5,6}$ They caution that the technology may be used not only to eliminate the mutations of life-threatening disorders but also to enhance "human capabilities" such as intelligence and appearance. ${ }^{6}$

The "good" news, though, is that genetically enhancing individuals is not that simple. Programming favorable traits in embryos may not be possible even with the most accurate and reliable version of CRISPR. Technology is not the limiting factor in the enhancement of individuals; nature is. A trait can be programmed in embryos only when the following two criteria are met.

First, the trait must be predominantly determined by DNA, which means that its heritability must be $100 \%$ or close to it. The lower the heritability, the greater the role of nongenetic factors such as lifestyle, education, and stress and the lower the likelihood that the presence of a trait can be guaranteed. Indeed, the heritability of most human traits is too limited for enhancing offspring through gene editing. The most heritable human trait, eye color, has an estimated heritability of $98 \%$, freckles has 91\%, and hair curliness has $85-95 \% .^{7}$ A recent review summarizing 50 years of heritability research showed that most potentially desirable human traits have much lower estimates: the heritability of intelligence and higher-level cognitive function is $\sim 50 \%$, muscle power $\sim 70 \%$, and temperament and personality $\sim 45 \%{ }^{8}$

Second, for enhancement to be practical, the traits in question must be caused by a single variant or an interaction among a limited number of variants. Although it may become technically possible to edit DNA accurately at multiple loci, it is unlikely that we will learn anytime soon what exactly needs to be programmed when tens or hundreds of variants are implicated. Moreover, gene editing for favorable traits is not just a matter of tweaking the relevant genes in the "right" direction. People are intelligent not because they have the "right genes" and had the "right environment" but because they have the right combination of genes and environment. Because the future environment of the embryo is unknown at the moment of editing, it will be impossible to know what the right genes need to be, now and in the future.

With these two criteria in mind, it is understandable why the first experiment using human embryo editing aimed to repair a mutation for $\beta$-thalassemia and not one for type 2 diabetes, depression, or even cancer. ${ }^{9} \beta$-Thalassemia is a recessive disorder caused by a single mutation. Other diseases that are mentioned as examples of future applications for gene editing, such as Duchenne muscular dystrophy and cystic fibrosis, are all single-gene recessive disorders. It is for these types of diseases that genome editing technologies hold promise for therapies and, potentially, for prevention by genome editing if embryo selection after preimplantation genetic diagnosis is not an option.

These criteria also explain why genome editing for intelligence, appearance, and other common polygenic traits and diseases should not be anticipated. Despite the successes in gene discovery over the past 10 years, the combined contribution of all genetic variants is too limited for embryo editing. Even when all genes and their complex interactions are completely understood, the genetic contribution to phenotype will be too limited to "program" traits in embryos-polygenic traits and diseases are just not genetic enough.

Caution is also warranted for genetic editing of embryos for single-gene disorders. Mutations, like polymorphisms, may

${ }^{1}$ Department of Epidemiology, Rollins School of Public Health, Emory University, Atlanta, Georgia, USA; ${ }^{2}$ Department of Clinical Genetics/EMGO Institute for Health and Care Research, Section Community Genetics, VU University Medical Center, Amsterdam, The Netherlands. Correspondence: A. Cecile J.W. Janssens (cecile.janssens@emory.edu) 
have so-called antagonistic pleiotropic effects, whereby expression of the gene increases the risk of one disease and decreases the risk of another. ${ }^{10,11}$ Well-known examples are the protective effects of carrying one mutated recessive copy of the sickle cell or $\beta$-thalassemia genes, which reduces the risk of fatal malaria. ${ }^{10,12}$

The international summit proposed allowing the use of gene editing technology in basic and preclinical research and, for clinical studies, allowing its use for modification of the DNA of somatic cells but not of germ-line DNA. ${ }^{6}$ This proposal was endorsed by the academies of sciences of the United States, the United Kingdom, and China and is in line with a recent recommendation of the UNESCO International Bioethics Committee. ${ }^{5}$ The clinical introduction of germ-line editing needs further investigation regarding the potential adverse effects of the technology as well as a broader discussion of the ethical and societal implications. This discussion is justified, the risks are real, but it should follow an inventory of what, ultimately, can and cannot be meaningfully edited in embryo DNA. The possibilities for germ-line gene editing are determined not only by the technical capabilities of CRISPR but also by the underlying biology of traits and diseases. The origins of polygenic diseases and traits are simultaneously too complex and not sufficiently genetic. This will limit the opportunities for disease prediction in adults and prevent the genetic enhancement of babies, regardless of very real ethical concerns.

Nevertheless, although it seems unlikely that parents will be willing to take unpredictable and unknown risks in an attempt to enhance their offspring when the success of the editing is so uncertain, there will also be parents who feel tempted to modify the DNA to increase the likelihood of a trait and reduce the likelihood of disease. Also, inevitably, there will be companies eager to "serve" them, even in the absence of valid opportunities. It is going to be crucial to hold the companies to accurate and truthful marketing and not allow exaggerated claims, and proper regulations must be in place before they enter the market to protect parents and their future offspring.

\section{DISCLOSURE}

The author declares no conflict of interest.

\section{REFERENCES}

1. Whitworth KM, Lee K, Benne JA, et al. Use of the CRISPR/Cas9 system to produce genetically engineered pigs from in vitro-derived oocytes and embryos. Biol Reprod 2014;91:78.

2. Li D, Qiu Z, Shao Y, et al. Heritable gene targeting in the mouse and rat using a CRISPR-Cas system. Nat Biotechnol 2013;31:681-683.

3. Doudna JA, Charpentier E. Genome editing. The new frontier of genome engineering with CRISPR-Cas9. Science 2014;346:1258096.

4. Ledford H. CRISPR, the disruptor. Nature 2015;522:20-24.

5. UNESCO International Bioethics Committee (IBC). Report of the IBC on Updating Its Reflection on the Human Genome and Human Rights. Paris, France, 2015.

6. Organizing Committee for the International Summit of Human Gene Editing. On Human Gene Editing: International Summit Statement. Washington, DC, 2015. http://www8.nationalacademies.org/onpinews/newsitem. aspx?RecordID=12032015a. Accessed 1 February 2016.

7. SNPedia. Heritability. Secondary Heritability. http://www.snpedia.com/index. php/Heritability. Accessed 1 February 2016.

8. Polderman TJ, Benyamin B, de Leeuw CA, et al. Meta-analysis of the heritability of human traits based on fifty years of twin studies. Nat Genet 2015;47: 702-709.

9. Liang $P, X u Y$, Zhang $X$, et al. CRISPR/Cas9-mediated gene editing in human tripronuclear zygotes. Protein Cell 2015;6:363-372.

10. Carter AJ, Nguyen AQ. Antagonistic pleiotropy as a widespread mechanism for the maintenance of polymorphic disease alleles. BMC Med Genet 2011; $12: 160$.

11. Wang K, Baldassano $\mathrm{R}$, Zhang $\mathrm{H}$, et al. Comparative genetic analysis of inflammatory bowel disease and type 1 diabetes implicates multiple loci with opposite effects. Hum Mol Genet 2010;19:2059-2067.

12. Aidoo M, Terlouw DJ, Kolczak MS, et al. Protective effects of the sickle cell gene against malaria morbidity and mortality. Lancet 2002;359:1311-1312. 